\title{
Detection and Characterization by Immunohistochemistry of the NGcGM3 Ganglioside in the Chicken Animal Model of Ovarian Cancer
}

\author{
Angel Casaco Parada ${ }^{1 *}$, Addy Gonzalez Palomo ${ }^{1}$, Dale Buchanan Hales ${ }^{2}$, Karen Hales ${ }^{2}$ and Luis Enrique Fernandez ${ }^{1}$ \\ ${ }^{1}$ Center for Molecular Immunology, Department of Biology System, Havana, Cuba \\ ${ }^{2}$ Southern Illinois University School of Medicine, Department of Life Science, Carbondale, USA
}

"Corresponding author: Angel Casaco Parada, Center for Molecular Immunology, Department of Biology System, P.O. Box 16040, Havana 11600, Cuba, Tel: (537) 27179333490; Fax: (537) 27206 44; E-mail: casaco@cim.sld.cu

Received date: November 16, 2017; Accepted date: December 08, 2017; Published date: December 15, 2017

Copyright: $\odot 2017$ Parada AC, et al. This is an open-access article distributed under the terms of the Creative Commons Attribution License, which permits unrestricted use, distribution, and reproduction in any medium, provided the original author and source are credited.

\begin{abstract}
In spite of ovarian cancer mortality rates have tended to level off and decrease during the last years in several countries, the disease is still the gynecological cancer with the highest death rate. The current therapies for ovarian cancer include surgery and chemotherapy based on platinum and a taxane. Nevertheless, these therapies are only efficient at the first stages of the disease and the treatments are lack of specificity, further contributing to the high mortality rates of ovarian cancer. The adult laying hen is a very interesting model for studying the development of the epithelial ovarian cancer since hen develops intense ovulation, spontaneous ovarian cancers and metabolic progression similar to women. It is known that normal chicken cells and human cells have no metabolic pathway for NeuGc biosynthesis due to a partial deletion in the gene that encodes CMP-Neu5Ac hydroxylase. Gangliosides are interesting type of molecules, different from proteins that have been identified as tumor antigens. Mammalian cells have these types of glycosphingolipids on their plasmatic membranes. Due to gangliosides are over-expressed in many tumors compared with the corresponding normal tissues, they are considered as very attractive compounds for immunotherapy, attributing to them a very important role in the growth and metastatic tumor processes. The aim of this study is to elucidate by immunohistochemistry the existence of NeuGc-GM3 in hen ovarian cancers. Slides with normal and cancer ovarian tissues from 3.5 years old chickens were incubated with the very specific antiNeuGcGM3 ganglioside 14F7 Mab for immunohistochemical studies. These studies showed that the 14F7 Mab immunorecognition was mainly evidenced in epithelial ovarian tumors and not in normal tissues. The presence of NeuGc-containing gangliosides in chicken and human epithelial ovarian cancers makes the chicken an interesting and relevant animal model for further experimental and translational investigations on ganglioside based immunotherapy.
\end{abstract}

Keywords: Chicken animal model; Ovarian cancer; NGcGM3 ganglioside; 14F7 monoclonal antibody; Immunohistochemestry

\section{Introduction}

In spite of during the last years, ovarian cancer mortality rates have tended to level off and decrease in several high-income European countries and North America, where rates were highest, the disease is still the gynecological cancer with the highest death rate. In these counties oral contraceptives have a long-term protective effect on ovarian cancer risk and were introduced earlier and used more frequently.

Unfortunately, pattern was inconsistent in Latin America, with a few declines in Argentina, Chile and Uruguay and other upward trends in Colombia, Cuba, Mexico and Venezuela [1,2]. The current therapies for ovarian cancer include surgery and chemotherapy based on platinum and a taxane [3]. Nevertheless, these therapies are only efficient at the first stages of the disease [4].

In addition, the treatments lack specificity, further contributing to the high mortality rates of ovarian cancer $[3,4]$.

On the other hand, it is very difficult an early diagnosis of the disease since local metastases in the peritoneal cavity produce minimal and unspecific symptoms and signs. Consequently, a high percent of ovarian cancers are diagnosed when compromise other organs [2,5]. In spite of the treatment for ovarian cancer is constantly improving, the disease is still the fifth cause of mortality for cancer in women.

Target therapies with active or passive manipulations of the immune system involving antibodies or cytotoxic effector cells against specific tumor antigens have the possibility to increase the efficacy and decrease the toxicity of new treatments [6]. Gangliosides are another interesting type of molecules, different from proteins that have been identified as tumor antigens [7]. Mammalian cells have these variable types of glycosphingolipids on their plasmatic membranes. Due to gangliosides are over-expressed in many tumors compared with the corresponding normal tissues, they are considered as very attractive compounds for immunotherapy, attributing to them a very important role in the growth and metastatic tumor processes and also inducing immunosuppression. They are composed by a ceramide portion, followed by at least two monosaccharide units, linked to one or more sialic acid (SA) residues. The most commonly found SA in most animal species is $\mathrm{N}$-acetylneuraminic acid (NAcNeu), less frequently found is $\mathrm{N}$-glycolylneuraminic acid ( $\mathrm{NGcNeu}$ ) [8].

CMP-N-acetylhydroxylase is the enzyme that converts the NAcNeu acid into NGcNeu acid. Chickens and humans have a partial depletion in the gene that encodes this enzyme, which causes the loss of the NGcNeu SA in normal tissues. Nevertheless, some human cancers express the $\mathrm{N}$-glycolylated gangliosides. Interestingly, high expression 
Citation: Parada AC, Palomo AG, Hales DB, Hales K, Fernandez LE (2017) Detection and Characterization by Immunohistochemistry of the NGcGM3 Ganglioside in the Chicken Animal Model of Ovarian Cancer. J Oncol Transl Res 3: 123. doi:10.4172/2476-2261.1000123

Page 2 of 3

of NGcGM3 ganglioside has been found in ovarian and breast human cancers [8-10].

Some in vitro and in vivo models of epithelial ovarian cancers (EOC), which are the most frequently ovarian cancer, have been developed; using in vitro cultures of human EOC; the fruit fly; the mouse and the hen animal models. These models have been used for studying the genetic and therapeutic aspects of this cancer disease, unfortunately, few characteristics of the disease are known in its early stages. Particularly, the adult laying hen is a very interesting model for studying the development of the EOC since hen develops intense ovulation, spontaneous EOC and metabolic progression similar to women [2].

Domestic laying hens have been genetically modified to almost produce an egg a day, this high repetitive ovulation and the monthly ovulatory in women have the consequence that they are among the few animal species that develop similar and spontaneous ovarian cancers [11]. It is known that normal chicken cells and human cells have no metabolic pathway for NeuGc biosynthesis due to a partial deletion in the gene that encodes CMP-Neu5Ac hydroxylase [12]. The expression of the $\mathrm{N}$-glycolylated gangliosides in many human cancers has been associated with its incorporation from different diets into a malignant tissue with an altered and accelerated metabolism [13].

The aim of this study is to elucidate by immunohistochemistry the existence of NeuGc-GM3 in hen ovarian cancers.

\section{Material and Methods}

\section{Tissue samples}

We obtained tissues from 3.5 years old commercial strains of White Leghorn laying hens that presented epithelial neoplastic lesions on the ovaries and from selected 3.5 years old commercial strains of White Leghorn laying hens that did not show the presence of neoplastic lesions, animals were reared at the University of Illinois at UrbanaChampaign (UIUC) Poultry Research Farm, USA. Tissues were preserved for pathological formalin fixation characterization. Fixed tissues were imbedded in paraffin wax, microtomed into $5 \mu \mathrm{m}$-thick sections and adhered to slides, and then stained with hematoxylin and eosin (H\&E). H\&E stained slides were assessed by one of the authors (K.D.).

Immunohistochemical (IHC) studies were performed at the Center for Molecular Immunology (CIM), Havana, Cuba.

Slides containing samples from normal and EOC tissues were maintained at room temperature until use, and then they were dewaxed in xylene, rehydrated in ethanol and endogenous peroxidase activity blocked as previously described [9].

\section{Monoclonal antibody}

The murine version of the $14 \mathrm{~F} 7 \mathrm{Mab}$ (IgG1), which is a very specific anti- NeuGcGM3 ganglioside antibody were used. This Mab was produced at the Center for Molecular Immunology, Havana, Cuba, as previously described [14].

Immunohistochemical staining. Then, slides from normal and neoplastic ovarian tissues were incubated during $1 \mathrm{~h}$ at room temperature in a humid chamber with the murine 14F7 Mab.

Slides containing samples from a colon adenocarcinoma were considered as positive controls.
Subsequently, slides were rinsed twice in in TBS and incubated with a rabbit anti-mouse IgG polyclonal antibody, washed for 10 minutes with TBS and then incubated with the avidin/biotin/peroxidase complex. We used the DAB substrate chromogen solution for visualizing the enzymatic activity.

For counterstaining slides the Mayer's Hematoxylin was used.

All reagents are from Dako, Carpinteria, USA.

\section{Evaluation of results}

A blind researcher (A.G.) evaluated qualitatively the intensity of the reaction in each sample as negative; weak; moderate or intense.

\section{Results}

The murine 14F7 Mab recognized 9 from 10 (90\%) of the epithelial ovarian tumors and 1 of the $8(12.5 \%)$ of the normal tissues. NeuGcGM3 staining was moderate in most ovarian cancer cases, while the intensity was weak in the only one normal ovarian tissues recognized by the murine 14F7 Mab.

In general, the 14F7 Mab recognized the ganglioside as a finely granular staining in cell membrane and cytoplasm of neoplastic cells.

Figure 1 shows an intense NGcGM3-positive immunostaining on the plasmatic membranes and the cytoplasms of chicken epithelial ovarian cancer cells.

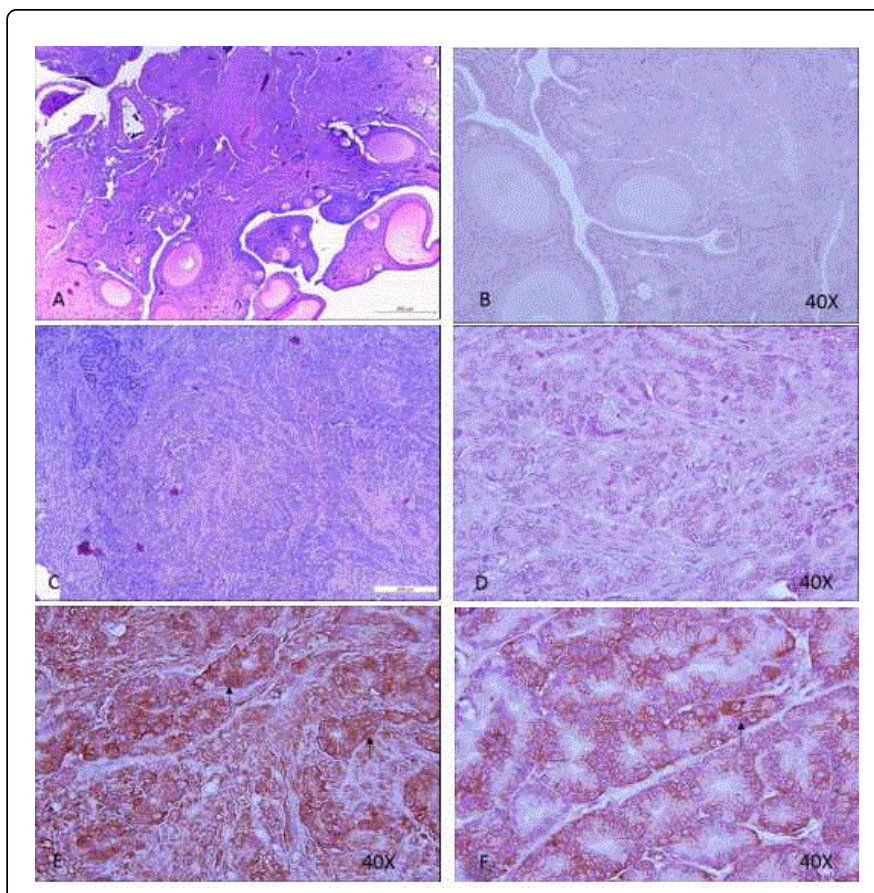

Figure 1: Hematoxylin and eosin staining in normal (A) and carcinoma (C) chicken ovarian tissues, NGcGM3-negative staining in ovarian normal tissue (B), An intense NGcGM3-positive Grade 3 immunostaining with $14 \mathrm{~F} 7 \mathrm{Mab}$ was located on both cell membrane and cytoplasm in malignant epithelial cells (E) and (F), (black arrows), Panel (D) shows the isotype control sample. 


\section{Discussion}

According to our knowledge, apart from Marek's disease, which is a contagious viral neoplastic disease in chickens [15], for the first time we report the expression of $\mathrm{N}$-glycolylated gangliosides in chicken ovarian tumors. The IHC study applying the very specific 14F7 Mab showed the presence of NeuGc-GM3 in $90 \%$ of the epithelial ovarian tumors.

One ovarian tissue from a normal hen expresses the NGcGM3, similar results have been observed by Rances et al. in normal human ovarian tissues [9]. The real possibility that NeuGc containing glycoconjugates were taken up from the chicken diet could be taken into account but it is improbable since the hen normal diet does not contained red meat.

Nevertheless, the role of alternative biosynthetic pathways of NeuGc in chicken or human neoplasia is probable but it is unknown [13].

The absence or low quantity of NeuGc-containing gangliosides in normal chicken and human tissues makes these gangliosides immunogenic.

In spite of there was a few number of studied cases, the present information of a specific neo-antigen in chicken ovarian tumor could be of value for the design of new immunotherapeutic protocols in the non-clinical setting but, a higher number of cases should be studied for reaffirming the present result. It would be also important to study the presence of the NGcGM3 ganglioside in others localizations of malignant tumors in chickens.

Some previous articles have reported the importance of the chicken animal model of ovarian cancer for studying the prevention of this disease.

Subcutaneous treatment of medroxyprogesterone acetate led to a reduction in the incidence of reproductive tract adenocarcinoma in hens [16], whereas the incidence of localized and metastatic ovarian cancers in hens treated with moderate or high doses of the p53 stabilizing compound CP-31398 was significantly lower than in nontreated hens [17].

It has been demonstrated that the diet supplemented with flaxseed reduces the severity of ovarian adenocarcinoma in hens [18].

Complementary bio-therapy with an anti-NGcGM3 cancer vaccine [19] or the anti-NGcGM3 ganglioside monoclonal antibody 14F7 [14] may reduce the need for high doses of the cytotoxic drugs used in current protocols or improve chemotherapy results.

Future studies with larger series chickens with ovarian and other tumor localizations, could correlate the immunohistochemical expression of NeuGc-GM3 with established clinical variables, staging, treatments, and diseases progression.

While previous non-clinical and clinical studies have clearly shown that NGcGM3 is a tumor-associated antigen with diagnostic and therapeutic potential in some human cancers $[10,19]$, the chicken animal model of ovarian cancer guarantee an interesting model for further experimental and translational investigations in this disease.

The laying hen could be an excellent model to study the effects of immunodetection and immunotherapeutic interventions on ovarian cancers (i.e. the naked 14F7 Mab; the labelled 14F7 Mab; and the NGcGM3 cancer vaccine).

\section{References}

1. Malvezzi M, Carioli G, Rodriguez T, Negri E, La Vecchia C (2016) Global trends and predictions in ovarian cancer mortality. Ann Oncol 27: 2017-2025.

2. Bernardo AM, Thorsteinsdottir S, Mummery CL (2015) Advantages of the avian model for human ovarian cancer. Mol Clin Oncol 3: 1191-1198.

3. Luvero D, Milani A, Ledermann JA (2014) Treatment options in recurrent ovarian cancer: latest evidence and clinical potential. Ther Adv Med Oncol 6: 229-239.

4. Menon U, Griffin M, Gentry-Maharaj A (2014) Ovarian cancer screening--current status, future directions. Gynecol Oncol 132: 490-495.

5. Goff BA, Mandel LS, Drescher CW, Urban N, Gough S, et al. (2007) Development of an ovarian cancer symptom index: possibilities for earlier detection. Cancer 110: 226-227.

6. Rosenberg SA (1999) A new era for cancer immunotherapy based on the genes that encode cancer antigens. Immunity 10: 281-287.

7. Lage A, Perez R, Fernandez LE (2005) Therapeutic cancer vaccines: at midway between immunology and pharmacology. Curr Cancer Drug Targets 5: 611-627.

8. Labrada M, Clavell M, Bebelagua Y, Leon Jd, Alonso DF, et al. (2010) Direct validation of NGcGM3 ganglioside as a new target for cancer immunotherapy. Expert Opin Biol Ther 10: 153-162.

9. Blanco R, Cedeño M, Escobar X, Blnco D, Rengifo CE, et al. (2011) Immunorecognition of the 14F7 Mab raised against N-glycolyl GM3 ganglioside in some normal and malignant tissues from genitourinary system. ISRN Pathol 953803.

10. Oliva JP, Valdes Z, Casaco A, Pimentel G, González J, et al. (2006) Clinical evidences of GM3 (NeuGc) ganglioside expression in human breast cancer using the 14F7 monoclonal antibody labelled with 99mTc. Breast Cancer Res Treat 96: 115-121.

11. Johnson PA, Stephens CS, Giles JR (2015) The domestic chicken: Causes and consequences of an egg a day. Poult Sci 94: 816-820.

12. Irie A, Suzuki A (1998) CMP-N-Acetylneuraminic acid hydroxylase is exclusively inactive in humans. Biochem Biophys Res Commun 248: 330-333.

13. Bardor M, Nguyen DH, Diaz S, Varki A (2005) Mechanism of uptake and incorporation of the non-human sialic acid N-glycolylneuraminic acid into human cells. J Biol Chem 280: 4228-4237.

14. Carr A, Mullet A, Mazorra Z, Vázquez AM, Alfonso M, et al. (2000) A mouse IgG1 monoclonal antibody specific for N-glycolyl GM3 ganglioside recognized breast and melanoma tumors. Hybridoma 19: 241-247.

15. Kawai T, Kato A, Higashi H, Kato S, Naiki M (1991) Quantitative determination of $\mathrm{N}$-glycolylneuraminic acid expression in human cancerous tissues and avian lymphoma cell lines as a tumor-associated sialic acid by gas chromatography-mass spectrometry. Cancer Res 51: 1242-1246.

16. Barnes MN, Berry WD, Straughn JM, Kirby TO, Leath CA, et al. (2002) A pilot study of ovarian cancer chemoprevention using medroxyprogesterone acetate in an avian model of spontaneous ovarian carcinogenesis. Gynecol Oncol 87: 57-63.

17. Mocka EH, Stern RA, Fletcher OJ (2017) Chemoprevention of spontaneous ovarian cancer in the domestic hen. Poult Sci 96: 1901-1909.

18. Dikshit A, Hales K, Hales DB (2017) Whole flaxseed diet alters estrogen metabolism to promote 2-methoxtestradiol-induced apoptosis in hen ovarian cancer. J Nutr Biochem 42: 117-25.

19. Fernandez LE, Gabri MR, Guthmann MD, Gomez RE, Gold S, et al. (2010) NGcGM3 ganglioside: a privileged target for cancer vaccines. Clin Dev Immunol 2010: 814397. 